Western North American Naturalist 68(2), (C) 2008, pp. 194-209

\title{
SPECIES COMPOSITION AND HABITAT ASSOCIATIONS OF BENTHIC ALGAL ASSEMBLAGES IN HEADWATER STREAMS OF THE SIERRA NEVADA, CALIFORNIA
}

\author{
Larry R. Brown ${ }^{1,2}$, Jason T. May ${ }^{1}$, and Carolyn T. Hunsaker ${ }^{3}$
}

\begin{abstract}
Despite their trophic importance and potential importance as bioindicators of stream condition, benthic algae have not been well studied in California. In particular there are few studies from small streams in the Sierra Nevada. The objective of this study was to determine the standing crop of chlorophyll- $a$ and benthic algal species assemblages present in the small 1st- and 2nd-order streams of the Kings River Experimental Watersheds (KREW, watersheds of Bull, Providence, Duff, and Teakettle Creeks) and determine the associations of these measures with stream habitat. We collected samples of benthic algae from rock substrata in September 2002 (7 sites) and 2005 (the same 7 sites plus 5 additional sites). Habitat and water-quality data were collected concurrently. Chlorophyll- $a$ values ranged from 0.2 to $3.2 \mathrm{mg} \cdot \mathrm{m}^{-2}$. Chlorophyll- $a$ in the Bull Creek watershed was generally lower than in the other watersheds. Benthic algal assemblages were dominated by diatoms and cyanobacteria. We collected 79 taxa of diatoms in 2002 and 126 taxa in 2005. Diatom taxa richness in individual samples ranged from 15 to 47 . Nonmetric multidimensional scaling analysis of arcsine square-root transformed proportional abundances of diatoms identified 3 groups of sites. Bull Creek sites were generally different from other sites (group 1), and the sites from Bull Creek were different in 2002 (group 2) and 2005 (group 3). Five taxa appeared to be particularly important in distinguishing groups: Achnanthidium minutissimum, Cocconeis placentula, Eunotia incisa, Eunotia pectinalis var. minor, and Planothidium lanceolatum. Elevation, water temperature, $\mathrm{pH}$, specific conductance, and canopy were habitat variables correlated with the differences in diatom assemblages among sites. Our results provide a valuable baseline for future studies of benthic algae in Sierra Nevada headwater streams and will be particularly important in understanding the effects of different forest restoration management strategies being tested in the KREW project.
\end{abstract}

Key words: benthic algae, California, chlorophyll-a, diatom assemblages, headwater streams, Sierra Nevada.

Periphyton respond directly to many aspects of the stream environment that might be expected to change with land management practices including nutrients (Chetelat et al. 1999, Francouer et al. 1999, Biggs 2000, Stelzer and Lamberti 2001), light (Wellnitz et al. 1996, Mosisch et al. 1999), and hydrology (Biggs and Hickey 1994, Biggs et al. 1999a,1999b). Because human activities modify stream habitats, monitoring of algal assemblages has been suggested as a method for assessing the effects of humans on streams (McCormick and Cairns 1994, Pan et al. 1996, Rott et al. 1998, Hill et al. 2000). Studies utilizing algae as indicators of environmental quality have been very successful at documenting differences in algal assemblages in relation to both natural and human-caused environmental gradients (Leland 1995, Kutka and Richards 1996, Carpenter and Waite 2000, Leland and Porter 2000, Sonneman et al. 2001, Weilhoefer and Pan 2006). Periphyton studies have also been successful at documenting effects of timber harvest practices (Shortreed and Stockner 1983, Kiffney and Bull 2000, Naymik and Pan 2005). Benthic algae not only serve as biological indicators, but are also the most successful primary producers in small streams and a food resource for many stream organisms, primarily macroinvertebrates. Thus, changes in periphyton production due to natural factors or human activities may affect the macroinvertebrate assemblage through the food web.

Despite their trophic importance and potential as bioindicators, benthic algae have not been well studied in California, especially in small streams. Recent studies of species composition have focused on larger rivers (Brown and Short 1999, Leland et al. 2001) or waterways at low elevations (Pan et al. 2006). In the Sierra Nevada, studies have focused primarily on lacustrine diatom assemblages as indicators

\footnotetext{
${ }^{1}$ U.S. Geological Survey, Placer Hall, 6000 J Street, Sacramento, CA 95819.

2E-mail: lrbrown@usgs.gov

${ }^{3}$ Sierra Nevada Research Center, Pacific Southwest Research Station, 2081 E. Sierra Avenue, Fresno, CA 93710
} 

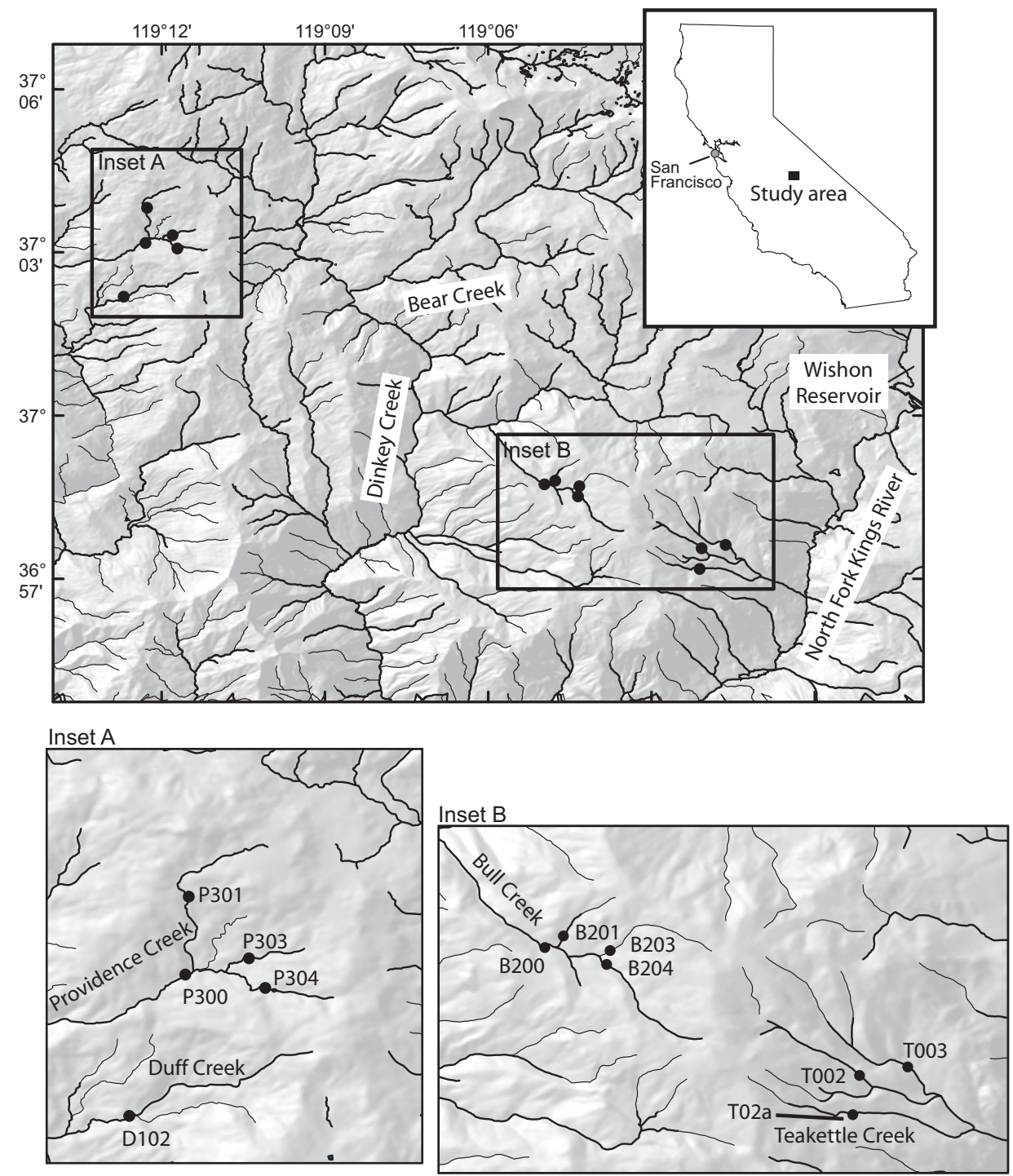

Fig. 1. Location of study sites in the Kings River Experimental Watersheds in September 2002 and 2005.

of long-term changes in $\mathrm{pH}$ or climate (Whiting et al. 1989, Bloom et al. 2003). A few studies have dealt with other aspects of algal ecology, including the effects of both acid mine drainage (Leland and Carter 1984, 1985) and recreational activities (Brown and Short 1999). The objective of this study was to determine chlorophyll- $a$ standing crop and benthic algal species assemblages present in the small 1st- and 2ndorder streams of the Kings River Experimental Watersheds (KREW) of the U.S. Department of Agriculture, Forest Service (USFS), and to determine the associations of these measures with stream habitat. These data will form a baseline for the assessment of changes in algal assemblages associated with forest restoration management practices (thinning and prescribed fire) in the experimental watersheds (Hunsaker and Egan 2003, http://www.fs.fed.us/psw/programs/snrc/water/kingsriver/).

\section{METHODS}

The research area is the 61,000-ha Kings River Sustainable Forest Ecosystem Project in 


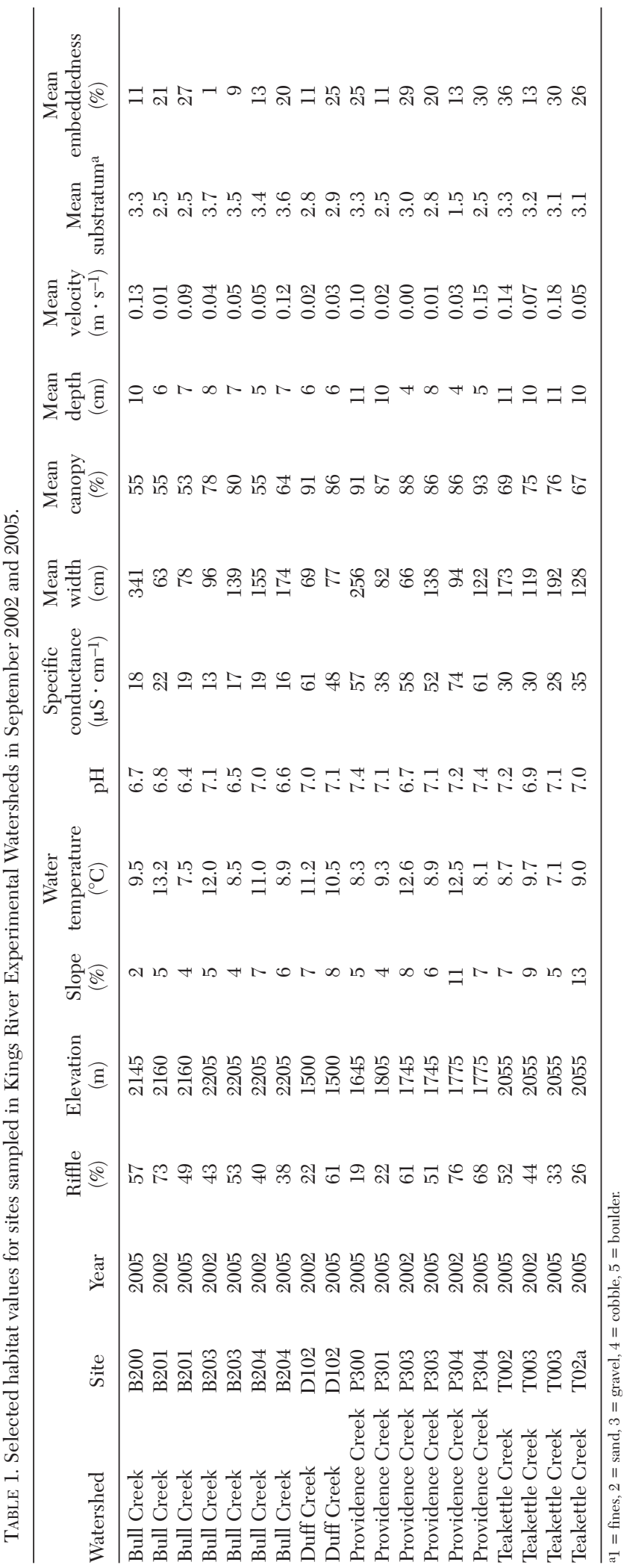


the Sierra National Forest of California (Fig. 1). The KREW study areas include the watersheds of Providence Creek, Duff Creek, Bull Creek, and Teakettle Creek (Fig. 1). Individual watersheds vary in area from 90 to $170 \mathrm{ha}$. The research area supports a mixed-conifer forest on granite-derived soils. Sierra National Forest fire records indicate that no fires have occurred in any of the research watersheds since 1911. The most recent timber harvesting occurred in the Providence Creek watershed between 1988 and 1992, and in the Bull Creek watershed between 1983 and 1993. Flows range from $0.001 \mathrm{~m}^{3} \cdot \mathrm{s}^{-1}$ in the summer to over 0.57 $\mathrm{m}^{3} \cdot \mathrm{s}^{-1}$ during rain-on-snow winter storms.

We conducted 2 surveys of algal assemblage structure, biomass (measured as chlorophyll- $a$ ), and habitat. We sampled 7 sites on 4-6 September 2002 and the same 7 sites plus 5 additional sites on 20-22 September 2005 (Table 1). An algal sample was collected from riffle habitat within a 100-m reach of stream at each site. Algal sampling was conducted according to standard U.S. Geological Survey (USGS) protocols (Moulton et al. 2002). At each site we collected 3-5 rocks at each of 5 locations within the study reach and brushed the exposed top surfaces of the rocks with a stiff brush to remove algae (top rock scrape). We then measured the brushed areas on each rock by cutting an aluminum foil template to the size of the sample area. The areas of the templates were determined in the laboratory using a digitizing tablet and then summed for each site to provide the total area sampled. All benthic algae were combined into a single composite sample.

Triplicate 5-mL subsamples were taken from the composite sample for determination of chlorophyll- $a$. Each 5-mL subsample was filtered onto a $47-\mathrm{mm}$ glass fiber filter. We removed macroinvertebrates, detritus, and large substratum particles with forceps, after which the filter was folded into quarters, wrapped in foil, placed in a labeled plastic bag, and frozen on dry ice. Samples were kept frozen in a freezer until analyzed. Chlorophyll- $a$ was determined fluorometrically following the methods in Wetzel and Likens (1978).

The remaining portion of the sample was analyzed for identification and enumeration of algae taxa. Soft-body algae (live nondiatom algae) were identified and enumerated in a Palmer Counting Chamber at 400X magnification using a microscope. We identified a total of
300 algal counting units (live soft-body algae and diatoms) and enumerated them at 400X magnification under the microscope. For colonial algae, each colony was counted as a single algal unit for purposes of tallying 300 counting units in a count. For thin, filamentous bluegreen algae in which cross-walls are often difficult to detect, we counted a 10- $\mu$ m length of trichome as 1 algal unit. Individual diatom cells were counted as 1 counting unit. Periphyton samples were processed with concentrated sulfuric acid and potassium dichromate for diatom analysis (Patrick and Reimer 1966). After numerous rinses with distilled water, cleaned diatom frustules were mounted in mounting medium to make permanent slides. A total of 600 diatom valves (300 diatom frustules or cells) were enumerated at 1000X magnification and identified to the species level whenever possible, using current taxonomic references. Chlorophyll- $a$ and identification and enumeration of algae were conducted at Portland State University, Oregon, under the direction of Dr. Yangdong Pan.

Personnel of the USDA Forest Service and the Sierra Nevada Aquatic Research Laboratory characterized habitat. The lengths $(\mathrm{m})$ of riffle and pool in the reach were measured with a fiberglass tape. Gradient was measured with a clinometer. At transects located every $10 \mathrm{~m}$ along the reach, the wetted width $(\mathrm{cm})$ was measured. Overhead canopy was measured with a densiometer at the center and edges of the stream at each transect. Depth $(\mathrm{cm})$, water velocity $\left(\mathrm{cm}^{\cdot} \mathrm{s}^{-1}\right)$, and substratum (fines, sand, gravel, cobble, boulder) were measured at 5 equally spaced points along each transect. Embeddedness of cobbles (\%) was estimated for cobbles in fines or sand. Cobbles on gravel or on other cobbles were not considered embedded. After all habitat measurements were completed, water temperature, conductivity, and $\mathrm{pH}$ were measured with electronic meters. Water samples were collected in 2005 for analyses of nitrate (Hautman et al. 1997) and phosphate (O’Dell et al. 1993) at the USDA Forest Service, Pacific Southwest Research Station Laboratory, Riverside, CA. Discharge was measured at permanent flumes for Duff Creek and 3 sites in the Providence Creek watershed in 2002. Discharge was available from the same 4 sites plus 3 sites in the Bull Creek watershed and 1 site in the Teakettle Creek watershed in 2005. 
TABLE 2. Mean chlorophyll- $a$ concentration $\left(\mathrm{mg} \cdot \mathrm{m}^{-2}\right)$ and diatom taxa richness for sites in the Kings River Experimental Watersheds in September 2002 and 2005. The standard deviation of chlorophyll- $a$ concentration (in parentheses) represents measurement error determined from 3 subsamples from a single composite sample.

\begin{tabular}{lccccc}
\hline & \multicolumn{2}{c}{$\begin{array}{c}\text { Chlorophyll- } a \\
\text { concentration }\end{array}$} & & \multicolumn{2}{c}{$\begin{array}{c}\text { Diatom taxa } \\
\text { richness }\end{array}$} \\
Site & 2002 & 2005 & & 2002 & 2005 \\
\hline B200 & & $0.5(0.1)$ & & 19 \\
B201 & $0.8(0.3)$ & $0.4(0.1)$ & & 31 & 36 \\
B203 & $0.7(0.3)$ & $0.6(0.1)$ & & 29 & 34 \\
B204 & $1.1(0.3)$ & $0.4(0.2)$ & & 15 & 47 \\
D102 & $3.2(1.0)$ & $1.8(0.2)$ & & 27 & 26 \\
P300 & & $0.9(0.5)$ & & 36 \\
P301 & & $1.7(0.1)$ & & 33 \\
P303 & $1.6(0.3)$ & $2.4(0.2)$ & & 34 & 41 \\
P304 & $0.2(0.1)$ & $0.9(0.1)$ & & 36 & 33 \\
T002 & & $1.2(0.1)$ & & 28 \\
T003 & $1.7(0.1)$ & $0.7(0.1)$ & & 36 & 40 \\
T02a & & $1.3(0.2)$ & & 45 \\
\hline
\end{tabular}

We summarized algal taxon data as percentages. Diatom data were analyzed in more detail. For abundant taxa, defined as $>5 \%$ of the assemblage at any site, we examined autecological data available from the literature to determine if these taxa were indicative of particular environmental conditions. Using nonmetric multidimensional scaling (NMS), we examined patterns in species assemblage. NMS is an ordination technique that summarizes (reduces the dimensionality of) a matrix of among-sample similarity coefficients. NMS is similar in concept to other ordination techniques, such as principal components analysis or correspondence analysis. Species proportional abundances were arcsine square-root transformed for analysis, and Bray-Curtis similarities were used as the similarity coefficient. The "fit" of the ordination is assessed by the stress value. Conceptually, stress compares the original similarities between samples calculated from the full data matrix with similarities calculated between samples in the lower-dimension ordination space. Stress of $<0.20$ indicates that the lower-dimension ordination successfully reproduces the patterns present in the higher-dimension ordination space (Clarke and Warwick 2001). We calculated Spearman correlations of the abundances of selected species with the NMS axes to better understand the ecological meaning of the axes.

By using the program SIMPER (Clarke and Warwick 2001), we could discern similarities within groups and differences between groups identified from NMS results (see results). The program determines mean similarities of samples within groups, the mean dissimilarity of samples between groups, and the percentage contribution of each species to the similarity or dissimilarity value of interest.

We used principal components analysis to characterize gradients in the habitat data. We included mean and coefficient of variation in the data analysis for variables with multiple measurements per site. As an indication of antecedent flow conditions, we summarized the discharge data for the 30 days prior to sampling. We tested for the associations of habitat variables with log-transformed mean chlorophyll- $a$ data by calculating Pearson correlations. Using the BV-STEP procedure in PRIMER (Clarke and Warwick 2001), we tested the correlation of diatom assemblage composition with environmental factors. This multivariate permutation test compares a matrix of Euclidean distances calculated from environmental measurements with the species' Bray-Curtis similarity matrix. A high correlation indicates a strong association of the species assemblage with the measured environmental variables. This analysis proceeds in a stepwise manner and tests combinations of environmental variables to determine the best model. This test was based on 100 permutations of the sample data.

\section{Results}

Chlorophyll- $a$ values ranged from $0.2 \mathrm{mg}$. $\mathrm{m}^{-2}$ to $3.2 \mathrm{mg} \cdot \mathrm{m}^{-2}$ (Table 2). Chlorophyll- $a$ was higher in 2002 than in 2005 in the Bull Creek, Duff, and Teakettle watersheds, but the opposite occurred in the Providence Creek watershed. Chlorophyll- $a$ values at sites in the Bull Creek watershed were lower than values at sites in the other watersheds in each year (Table 2), except for 1 site in the Providence Creek watershed in 2002.

Benthic algal assemblages were dominated by diatoms (Bacillariophyta) and cyanobacteria (Cyanophyta, blue-green algae; Table 3). The major exception to this pattern was site B204 in 2002, where there was a relatively equal balance among diatoms, cyanobacteria, and green algae. The cyanobacteria Anabaena and Oscillatoria were particularly common (Table 4) in both years. Green algae (Chlorophyta) were present at most sites in smaller percentages 
TABLE 3. Percentages of different algal groups collected from sites in the Kings River Experimental Watersheds in September 2002 and 2005. Total count is the total number of algae identified for calculation of percentages.

\begin{tabular}{|c|c|c|c|c|c|c|c|}
\hline Site & Year & $\begin{array}{l}\text { Total } \\
\text { count }\end{array}$ & Diatoms & $\begin{array}{l}\text { Green } \\
\text { algae }\end{array}$ & $\begin{array}{c}\text { Cyano- } \\
\text { bacteria }\end{array}$ & $\begin{array}{l}\text { Red } \\
\text { algae }\end{array}$ & $\begin{array}{l}\text { Yellow-green } \\
\text { algae }\end{array}$ \\
\hline B200 & 2005 & 321 & 54.7 & 0 & 43.2 & 2.1 & 0 \\
\hline B201 & 2002 & 462 & 28.1 & 2.3 & 58.6 & 11.1 & 0 \\
\hline B201 & 2005 & 450 & 31.2 & 0.9 & 67.9 & 0 & 0 \\
\hline B203 & 2002 & 431 & 55.6 & 8.7 & 33.8 & 1.9 & 0 \\
\hline B203 & 2005 & 331 & 52.0 & 2.6 & 45.3 & 0 & 0 \\
\hline B204 & 2002 & 492 & 33.4 & 36.0 & 30.6 & 0 & 0 \\
\hline B204 & 2005 & 387 & 37.3 & 0.3 & 59.0 & 3.4 & 0 \\
\hline D201 & 2002 & 632 & 51.2 & 0.2 & 48.6 & 0 & 0 \\
\hline D201 & 2005 & 541 & 93.7 & 0 & 6.3 & 0 & 0 \\
\hline P300 & 2005 & 402 & 49.0 & 0 & 51.0 & 0 & 0 \\
\hline P301 & 2005 & 319 & 72.6 & 0 & 27.4 & 0 & 0 \\
\hline P303 & 2002 & 531 & 78.6 & 1.9 & 19.5 & 0 & 0 \\
\hline P303 & 2005 & 562 & 61.8 & 2.9 & 28.7 & 0.2 & 6.4 \\
\hline P304 & 2002 & 468 & 36.2 & 4.9 & 58.9 & 0 & 0 \\
\hline P304 & 2005 & 545 & 44.5 & 0 & 55.5 & 0 & 0 \\
\hline T002 & 2005 & 404 & 89.4 & 0 & 10.6 & 0 & 0 \\
\hline T003 & 2002 & 670 & 89.4 & 8.7 & 1.9 & 0 & 0 \\
\hline Т003 & 2005 & 369 & 70.0 & 0.2 & 29.7 & 0 & 0 \\
\hline T02a & 2005 & 730 & 68.4 & 1.7 & 29.9 & 0 & 0 \\
\hline
\end{tabular}

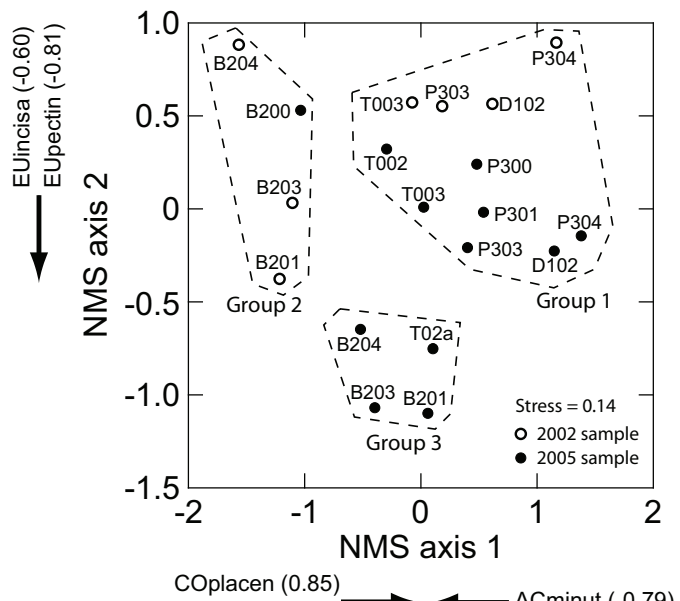

PLlanceo (0.80)

Fig. 2. Ordination plot of sample scores on the first 2 axes of a nonmetric multidimensional scaling analysis of diatom proportional abundances. Spearman rank correlations (all $P<0.05$ ) of proportional abundances of 5 important diatom species with ordination scores are shown in parentheses. Arrows indicate direction of increasing diatom abundances. Species abbreviations are ACminut, Achnanthidium minutissimum; COplacen, Cocconeis placentula; PLlanceo, Planothidium lanceolatum; EUincisa, Eunotia incisa; and EUpectin, Eunotia pectinalis var. minor. See Figure 1 for site locations.

(Table 3). Closterium was the most common taxon of green algae and was found during both years (Table 4). The only other taxon collected both years was Spirogyra (Table 4). Red algae
(Rhodophyta) occurred in 5 samples (Table 3), 4 of which were from the Bull Creek watershed. The only taxon of red algae collected was Audouinella (Table 4). Yellow-green algae (Xanthophyta) were found in 1 sample from the Providence Creek watershed in 2005. The only taxon present was Bumilleriopsis (Table 4).

We collected 79 taxa of diatoms in 2002 and 126 in 2005 (Table 5). Only 50 taxa were found in both years. Of the taxa collected in 2002, 34 taxa $(43 \%)$ were found only in 1 sample. Of the taxa collected in 2005, 41 taxa (33\%) were found only in 1 sample. Taxon richness of diatoms (number of diatom taxa) at individual sites varied from 15 to 36 in 2002 and from 19 to 47 in 2005 (Table 2). Only a few diatom taxa were abundant. In 2002, only 21 taxa constituted more than $5 \%$ of the assemblage at any site (Table 4). In 2005 the number decreased to 19 taxa.

Of the 29 diatoms considered abundant in 1 or both years (Table 6), all but 5 (Aulacoseira alpigena, A. crenulata, A. distans, Fragilaria crotonensis, and Tabellaria flocculosa) are primarily benthic (Porter 2008). Of the 26 benthic species, only 4 are motile (Geissleria paludosa, Mayamaea atomus, Navicula cryptotenella, and Nitzschia linearis; Lowe 2003, Porter 2008). Although autecological data were not available for every species, the abundant diatoms were generally sensitive to organic pollution and indicative of high dissolved oxygen, low specific 
TABLE 4. Number of sites ( $n$, total number of sites sampled) where nondiatom taxa were collected and the maximum percentage of each taxa (based on total algae cells counted) in the samples for sites in the Kings River Experimental Watersheds in September 2002 and 2005.

\begin{tabular}{|c|c|c|c|c|}
\hline & \multicolumn{2}{|c|}{2002} & \multicolumn{2}{|c|}{2005} \\
\hline & $\begin{array}{l}\text { Count } \\
(n=7)\end{array}$ & $\begin{array}{c}\text { Maximum } \\
(\%)\end{array}$ & $\begin{array}{l}\text { Count } \\
(n=12)\end{array}$ & $\begin{array}{c}\text { Maximum } \\
(\%)\end{array}$ \\
\hline \multicolumn{5}{|l|}{ CYANOBACTERIA } \\
\hline Anabaena & 6 & 48.81 & 9 & 51.07 \\
\hline Calothrix & 4 & 4.33 & 0 & 0 \\
\hline Chamaesiphon & 1 & 1.71 & 0 & 0 \\
\hline Chroococcus & 4 & 1.5 & 0 & 0 \\
\hline Homeothrix & 0 & 0 & 1 & 2.44 \\
\hline Lyngbya & 7 & 13.92 & 0 & 0 \\
\hline Nostoc & 0 & 0 & 5 & 11.74 \\
\hline Oscillatoria & 7 & 39.43 & 9 & 39.93 \\
\hline Phormidium & 0 & 0 & 2 & 5.17 \\
\hline Spirulina & 0 & 0 & 2 & 0.25 \\
\hline Tolypothrix & 5 & 44.94 & 1 & 21.92 \\
\hline \multicolumn{5}{|l|}{ GREEN ALGAE } \\
\hline Ankistrodesmus & 1 & 1.08 & 0 & 0 \\
\hline Chlorella & 0 & 0 & 1 & 0.75 \\
\hline Closterium & 4 & 0.46 & 2 & 0.27 \\
\hline Cosmarium & 3 & 0.65 & 0 & 0 \\
\hline Kirchneriella & 0 & 0 & 1 & 0.18 \\
\hline Microspora & 0 & 0 & 1 & 8.72 \\
\hline Pediastrum & 1 & 0.22 & 0 & 0 \\
\hline Scenedesmus & 0 & 0 & 2 & 4.93 \\
\hline Spirogyra & 2 & 0.43 & 1 & 0.67 \\
\hline Staurastrum & 1 & 0.2 & 0 & 0 \\
\hline Ulothrix & 0 & 0 & 2 & 2.22 \\
\hline Unicellular spores & 5 & 35.5 & 0 & 0 \\
\hline \multicolumn{5}{|l|}{ RED ALGAE } \\
\hline Audouinella & 4 & 11.11 & 1 & 0.22 \\
\hline \multicolumn{5}{|l|}{ YELLOW-GREEN ALGAE } \\
\hline Bumilleriopsis & 0 & 0 & 1 & 6.44 \\
\hline
\end{tabular}

conductance, and low dissolved chloride (Table 6). The abundant species represented a wide range of $\mathrm{pH}$ optima, nutrient optima, and trophic conditions (Table 6).

A 2-dimensional NMS ordination adequately summarized the data (stress $=0.14$; Fig. 2). The ordination identified 3 large groups based on a minimum of $30 \%$ similarity among samples. The ordination mainly separates the Bull Creek samples from the other sites (group 1). The Bull Creek samples are then largely divided into groups based on year of sampling (2002, group 2 and 2005, group 3). Group 1 also showed differences among years with 2002 samples scoring higher on NMS axis 2.

The SIMPER (Clarke and Warwick 2001) results helped clarify similarities within groups and differences between groups. Average similarity among samples in group 1 was $41 \%$.
This group was characterized by similar percentages of Cocconeis placentula, Planothidium lanceolatum, Gomphosphenia spp., Gomphonma kobayasii, Navicula cryptotenella, and Achnanthidium minutissimum (taxa listed in decreasing order of importance). Average similarity among samples in group 2 was 39\%. Group 2 was characterized by similar percentages of Achnanthidium minutissimum, Fragilaria capucina, Diatoma mesodon, and Achnanthes nodosa. Average similarity among samples in group 3 was 47\%. Group 3 was characterized by Eunotia pectinalis var. minor, E. incisa, Cocconeis placentula, Achnanthidium minutissimum, E. implicata, and Gomphonema parvulum.

Average dissimilarity between samples in the different groups exceeded $70 \%$ for all group comparisons. Five taxa appeared to be particularly important in distinguishing groupsAchnanthidium minutissimum, Cocconeis placentula, Eunotia incisa, E. pectinalis var. minor, and Planothidium lanceolatum. Spearman correlations of species abundances with NMS axis scores showed a gradient of decreasing percentages of Achnanthidium minutissimum and increasing percentages of Cocconeis placentula and Planothidium lanceolatum along NMS axis 1 (Fig. 2). Samples from the Providence Creek and Duff Creek watersheds had low percentages of Achnanthidium minutissimum and high percentages of Cocconeis placentula and Planothidium lanceolatum compared with samples from the Bull Creek watershed. Teakettle Creek watershed samples were intermediate. NMS axis 2 shows a gradient in Eunotia species with high percentages of these species in group 3 samples.

In general, all sites were small, cool, and shaded, with mean substratum size ranging from fines to gravel (Table 1). Values for $\mathrm{pH}$ ranged from 6.4 to 7.4 , and specific conductance was low $\left(<74 \mu \mathrm{S} \cdot \mathrm{cm}^{-1}\right)$. Both nitrate and phosphate were below the method detection limit of $0.050 \mathrm{mg} \cdot \mathrm{L}^{-1}$ (nitrate as $\mathrm{N}$ and phosphate as $\mathrm{P}$, respectively) at all but 1 site each. Nitrate was $0.079 \mathrm{mg} \cdot \mathrm{L}^{-1}$ as $\mathrm{N}$ at site B204, and phosphate was $0.105 \mathrm{mg} \cdot \mathrm{L}^{-1}$ as $\mathrm{P}$ at site P300 in 2005.

The principal components analysis resulted in 6 axes with eigenvalues $>1$ that explained $82 \%$ of the variance in habitat data; however, the first 3 axes accounted for the majority of variance (68\%). PCA axis 1 identified a combined stream size and stream elevation gradient. Sites 
TABLE 5. Number of sites ( $n$, total number of sites sampled) where diatom taxa were collected and the maximum percentage of each diatom taxa (based on total diatoms counted) in the samples for sites in the Kings River Experimental Watersheds in September 2002 and 2005.

\begin{tabular}{|c|c|c|c|c|}
\hline \multirow[b]{2}{*}{ Scientific name } & \multicolumn{2}{|c|}{2002} & \multicolumn{2}{|c|}{2005} \\
\hline & $\begin{array}{l}\text { Count } \\
(n=7)\end{array}$ & $\underset{(\%)}{\operatorname{Maximum}}$ & $\begin{array}{c}\text { Count } \\
(n=12)\end{array}$ & $\begin{array}{c}\text { Maximum } \\
(\%)\end{array}$ \\
\hline Achnanthes nitidiformis & 1 & 1.95 & 0 & 0.00 \\
\hline Achnanthes nodosa & 5 & 8.29 & 9 & 6.17 \\
\hline Achnanthes oblongella & 1 & 3.41 & 1 & 0.33 \\
\hline Achnanthes pseudoswazi & 0 & 0.00 & 1 & 0.50 \\
\hline Achnanthidium catenatum & 1 & 0.17 & 0 & 0.00 \\
\hline Achnanthidium exiguum & 4 & 0.98 & 4 & 2.00 \\
\hline Achnanthidium minutissimum & 7 & 87.11 & 11 & 79.50 \\
\hline Achnanthidium pyrenaicum & 3 & 7.20 & 7 & 4.00 \\
\hline Amphipleura pellucida & 0 & 0.00 & 2 & 0.17 \\
\hline Amphora copulata & 0 & 0.00 & 3 & 0.50 \\
\hline Amphora ovalis & 3 & 0.99 & 0 & 0.00 \\
\hline Amphora pediculus & 0 & 0.00 & 3 & 0.33 \\
\hline Anomoeoneis brachysira & 2 & 0.66 & 0 & 0.00 \\
\hline Asterionella formosa & 1 & 0.16 & 0 & 0.00 \\
\hline Aulacoseira alpigena & 1 & 3.39 & 8 & 5.17 \\
\hline Aulacoseira ambigua & 0 & 0.00 & 1 & 1.00 \\
\hline Aulacoseira crenulata & 5 & 10.64 & 8 & 2.00 \\
\hline Aulacoseira distans & 5 & 6.99 & 2 & 0.33 \\
\hline Caloneis bacillum & 0 & 0.00 & 4 & 1.00 \\
\hline Chamaepinnularia spp. & 0 & 0.00 & 1 & 0.17 \\
\hline Cocconeis neodiminuta & 0 & 0.00 & 2 & 0.33 \\
\hline Cocconeis placentula & 4 & 13.63 & 12 & 58.67 \\
\hline Cyclotella meneghiniana & 1 & 0.16 & 0 & 0.00 \\
\hline Cymbella cistula & 0 & 0.00 & 1 & 0.33 \\
\hline Cymbella gracilis & 3 & 0.49 & 4 & 2.17 \\
\hline Cymbella naviculiformis & 0 & 0.00 & 1 & 0.33 \\
\hline Decussata placenta & 0 & 0.00 & 1 & 0.33 \\
\hline Diadesmis confervacea & 0 & 0.00 & 2 & 2.17 \\
\hline Diadesmis contenta & 3 & 1.66 & 0 & 0.00 \\
\hline Diadesmis gallica & 0 & 0.00 & 2 & 0.33 \\
\hline Diatoma anceps & 0 & 0.00 & 3 & 2.17 \\
\hline Diatoma mesodon & 7 & 2.42 & 9 & 11.50 \\
\hline Diatoma moniliformis & 0 & 0.00 & 1 & 0.33 \\
\hline Diploneis elliptica & 1 & 0.16 & 0 & 0.00 \\
\hline Diploneis oblongella & 0 & 0.00 & 1 & 0.33 \\
\hline Diploneis puella & 0 & 0.00 & 1 & 0.33 \\
\hline Encyопета minutum & 1 & 0.32 & 2 & 0.83 \\
\hline Encyonema muelleri & 0 & 0.00 & 2 & 0.50 \\
\hline Encyonema silesiacum & 0 & 0.00 & 4 & 1.83 \\
\hline Eolimna minima & 4 & 4.32 & 6 & 1.83 \\
\hline Epithemia adnata & 0 & 0.00 & 2 & 2.00 \\
\hline Epithemia sorex & 0 & 0.00 & 4 & 2.17 \\
\hline Epithemia turgida & 1 & 1.63 & 1 & 5.17 \\
\hline Eunotia bilunaris & 1 & 0.16 & 0 & 0.00 \\
\hline Eunotia curvata & 0 & 0.00 & 4 & 4.00 \\
\hline Eunotia formica & 2 & 0.33 & 1 & 3.50 \\
\hline Eunotia glacialis & 0 & 0.00 & 6 & 2.50 \\
\hline Eunotia implicata & 1 & 1.94 & 6 & 2.67 \\
\hline Eunotia incisa & 6 & 19.84 & 8 & 57.00 \\
\hline Eunotia monodon & 0 & 0.00 & 1 & 0.33 \\
\hline Eunotia muscicola & 2 & 4.91 & 0 & 0.00 \\
\hline Eunotia musicola var. tridentula & 0 & 0.00 & 3 & 6.50 \\
\hline Eunotia paludosa & 1 & 0.65 & 0 & 0.00 \\
\hline Eunotia pectinalis & 0 & 0.00 & 3 & 1.83 \\
\hline Eunotia pectinalis var. minor & 2 & 2.72 & 12 & 54.33 \\
\hline
\end{tabular}


TABLE 5. Continued.

\begin{tabular}{|c|c|c|c|c|}
\hline \multirow[b]{2}{*}{ Scientific name } & \multicolumn{2}{|c|}{2002} & \multicolumn{2}{|c|}{2005} \\
\hline & $\begin{array}{l}\text { Count } \\
(n=7)\end{array}$ & $\underset{(\%)}{\operatorname{Maximum}}$ & $\begin{array}{c}\text { Count } \\
(n=12)\end{array}$ & $\begin{array}{l}\text { Maximum } \\
(\%)\end{array}$ \\
\hline Eunotia pirla & 0 & 0.00 & 3 & 1.00 \\
\hline Eunotia serra & 0 & 0.00 & 2 & 0.17 \\
\hline Eunotia soleirolii & 0 & 0.00 & 1 & 3.17 \\
\hline Eunotia spp. & 0 & 0.00 & 1 & 0.33 \\
\hline Fallacia indifferens & 1 & 0.17 & 0 & 0.00 \\
\hline Fragilaria capucina & 6 & 20.48 & 10 & 3.33 \\
\hline Fragilaria capucina var. amphicephala & $a$ & 0.17 & 0 & 0.00 \\
\hline Fragilaria capucina var. capitellata & 0 & 0.00 & 1 & 0.83 \\
\hline Fragilaria capucina var. gracilis & 1 & 10.82 & 6 & 1.33 \\
\hline Fragilaria capucina var. rumpens & 0 & 0.00 & 4 & 4.17 \\
\hline Fragilaria crotonensis & 2 & 8.00 & 1 & 0.67 \\
\hline Fragilaria vaucheriae & 1 & 0.17 & 0 & 0.00 \\
\hline Fragilariforma bicapitata & 0 & 0.00 & 1 & 0.67 \\
\hline Frustulia rhomboides & 4 & 0.65 & 2 & 0.50 \\
\hline Frustulia vulgaris & 0 & 0.00 & 2 & 0.50 \\
\hline Geissleria acceptata & 0 & 0.00 & 5 & 3.67 \\
\hline Geissleria paludosa & 4 & 11.46 & 0 & 0.00 \\
\hline Gomphonema affine & 2 & 0.81 & 2 & 0.67 \\
\hline Gomphonema angustatum & 1 & 0.33 & 0 & 0.00 \\
\hline Gomphonema clavatum & 3 & 7.04 & 1 & 1.67 \\
\hline Gomphonema gracile & 4 & 1.15 & 3 & 0.67 \\
\hline Gomphonema intricatum & 1 & 1.33 & 0 & 0.00 \\
\hline Gomphonema kobayasii & 3 & 8.21 & 8 & 11.83 \\
\hline Gomphonema micropus & 0 & 0.00 & 2 & 0.33 \\
\hline Gomphonema minutum & 6 & 6.81 & 7 & 2.33 \\
\hline Gomphonema olivaceum & 0 & 0.00 & 1 & 0.33 \\
\hline Gomphonema parvulum & 4 & 2.24 & 12 & 7.50 \\
\hline Gomphonema pseudotenellum & 0 & 0.00 & 2 & 1.33 \\
\hline Gomphonema pumilum & 0 & 0.00 & 10 & 21.50 \\
\hline Gomphonema rhombicum & 6 & 2.56 & 5 & 10.50 \\
\hline Gomphonema spp. & 0 & 0.00 & 2 & 0.33 \\
\hline Gomphonema subclavatum & 0 & 0.00 & 4 & 1.33 \\
\hline Gomphonema truncatum & 0 & 0.00 & 5 & 1.00 \\
\hline Gomphosphenia spp. & 6 & 37.77 & 8 & 23.33 \\
\hline Hannaea arcus & 0 & 0.00 & 1 & 0.83 \\
\hline Karayevia laterostrata & 1 & 5.53 & 2 & 0.67 \\
\hline Kolbesia suchlandtii & 4 & 10.08 & 6 & 4.17 \\
\hline Luticola goeppertiana & 0 & 0.00 & 1 & 0.17 \\
\hline Mayamaea agrestis & 0 & 0.00 & 1 & 0.17 \\
\hline Mayamaea atomus & 3 & 8.47 & 2 & 5.17 \\
\hline Melosia varians & 0 & 0.00 & 5 & 3.00 \\
\hline Meridion circulare & 3 & 0.98 & 3 & 0.50 \\
\hline Meridion circulare var. constrictum & 0 & 0.00 & 3 & 1.00 \\
\hline Navicula angusta & 0 & 0.00 & 3 & 0.67 \\
\hline Navicula antonii & 0 & 0.00 & 1 & 0.17 \\
\hline Navicula bremensis & 1 & 0.17 & 0 & 0.00 \\
\hline Navicula capitatoradiata & 0 & 0.00 & 2 & 0.67 \\
\hline Navicula concentrica & 0 & 0.00 & 2 & 0.33 \\
\hline Navicula cryptocephala & 6 & 2.11 & 1 & 0.33 \\
\hline Navicula cryptotenella & 4 & 8.64 & 8 & 2.33 \\
\hline Navicula exilis & 0 & 0.00 & 2 & 0.50 \\
\hline Navicula incertata & 0 & 0.00 & 3 & 1.17 \\
\hline Navicula leptostriata & 1 & 0.80 & 0 & 0.00 \\
\hline Navicula lundii & 0 & 0.00 & 2 & 0.50 \\
\hline Navicula porifera & 1 & 0.33 & 0 & 0.00 \\
\hline Navicula radiosa & 1 & 0.33 & 6 & 0.83 \\
\hline Navicula spp. & 0 & 0.00 & 2 & 0.33 \\
\hline
\end{tabular}


TABLE 5. Continued.

\begin{tabular}{|c|c|c|c|c|}
\hline \multirow[b]{2}{*}{ Scientific name } & \multicolumn{2}{|c|}{2002} & \multicolumn{2}{|c|}{2005} \\
\hline & $\begin{array}{l}\text { Count } \\
(n=7)\end{array}$ & $\begin{array}{c}\text { Maximum } \\
(\%)\end{array}$ & $\begin{array}{c}\text { Count } \\
(n=12)\end{array}$ & $\begin{array}{c}\text { Maximum } \\
(\%)\end{array}$ \\
\hline Navicula subminuscula & 1 & 1.16 & 0 & 0.00 \\
\hline Navicula tenelloides & 2 & 1.16 & 2 & 0.33 \\
\hline Navicula tridentula & 3 & 0.33 & 1 & 0.33 \\
\hline Navicula trivialis & 0 & 0.00 & 1 & 0.50 \\
\hline Navicula veneta & 0 & 0.00 & 2 & 0.33 \\
\hline Neidium ampliatum & 0 & 0.00 & 3 & 0.33 \\
\hline Nitzschia communis & 1 & 0.33 & 0 & 0.00 \\
\hline Nitzschia dissipata & 0 & 0.00 & 3 & 4.00 \\
\hline Nitzschia fonticola & 0 & 0.00 & 3 & 0.67 \\
\hline Nitzschia frustulum & 2 & 0.66 & 1 & 0.33 \\
\hline Nitzschia linearis & 4 & 0.50 & 2 & 5.83 \\
\hline Nitzschia palea & 0 & 0.00 & 1 & 0.33 \\
\hline Nitzschia recta & 4 & 0.65 & 0 & 0.00 \\
\hline Nitzschia subacicularis & 1 & 0.96 & 0 & 0.00 \\
\hline Nupela lapidosa & 0 & 0.00 & 1 & 0.17 \\
\hline Pinnularia borealis & 0 & 0.00 & 1 & 0.33 \\
\hline Pinnularia divergentissima & 2 & 1.66 & 0 & 0.00 \\
\hline Pinnularia gibba & 1 & 0.32 & 0 & 0.00 \\
\hline Pinnularia maior & 0 & 0.00 & 1 & 0.33 \\
\hline Pinnularia microstauron & 0 & 0.00 & 2 & 0.50 \\
\hline Pinnularia subcapitata & 1 & 0.16 & 0 & 0.00 \\
\hline Pinnularia viridis & 0 & 0.00 & 1 & 0.33 \\
\hline Planothidium lanceolatum & 6 & 22.92 & 11 & 49.83 \\
\hline Psammothidium bioretii & 1 & 1.45 & 2 & 0.67 \\
\hline Psammothidium subatomoides & 0 & 0.00 & 4 & 1.67 \\
\hline Psammothidium ventralis & 0 & 0.00 & 1 & 0.33 \\
\hline Reimeria sinuata & 1 & 0.33 & 4 & 0.67 \\
\hline Rhoicosphenia abbreviata & 3 & 9.30 & 6 & 2.83 \\
\hline Rhopalodia gibba & 0 & 0.00 & 1 & 0.33 \\
\hline Sellaphora pupula & 0 & 0.00 & 3 & 0.83 \\
\hline Sellaphora seminulum & 1 & 0.17 & 1 & 0.33 \\
\hline Stauroneis anceps & 0 & 0.00 & 1 & 0.33 \\
\hline Stauroneis kriegeri & 0 & 0.00 & 2 & 0.33 \\
\hline Stauroneis phoenicenteron & 0 & 0.00 & 1 & 0.33 \\
\hline Stauroneis thermicola & 0 & 0.00 & 2 & 0.67 \\
\hline Staurosira construens & 4 & 4.20 & 3 & 5.17 \\
\hline Staurosirella pinnata & 1 & 0.16 & 5 & 1.17 \\
\hline Surirella elegans & 1 & 0.16 & 0 & 0.00 \\
\hline Surirella spp. & 0 & 0.00 & 1 & 0.17 \\
\hline Synedra delicatissima & 1 & 0.82 & 0 & 0.00 \\
\hline Synedra goulardi & 0 & 0.00 & 1 & 0.33 \\
\hline Synedra ulna & 4 & 4.13 & 6 & 6.83 \\
\hline Tabellaria flocculosa & 3 & 21.81 & 1 & 0.33 \\
\hline
\end{tabular}

in the Providence Creek and Duff Creek watersheds were at lower elevations and tended to be narrower with less variation in canopy, lower velocities, and smaller substrata than sites in the Bull Creek watershed. Teakettle Creek watershed sites were similar to Bull Creek sites (Fig. 3). PCA axis 2 mainly separated Bull Creek sites from the other sites. Bull Creek sites were lower in $\mathrm{pH}$, warmer, lower in velocity, and had lower percentages of embeddedness. PCA axis 3 did not separate watersheds or particular groups of sites. This axis mainly seemed to separate sites on the basis of amount of riffle habitat, variation in canopy, percentage embeddedness, and depth variation.

Stream discharge during the 30 days before sampling was generally higher in 2005 than in 2002 (Table 7). Discharge was from 2.6 to 7.7 times higher in 2005 compared to 2002 at the 4 sites with data for both years. Teakettle 


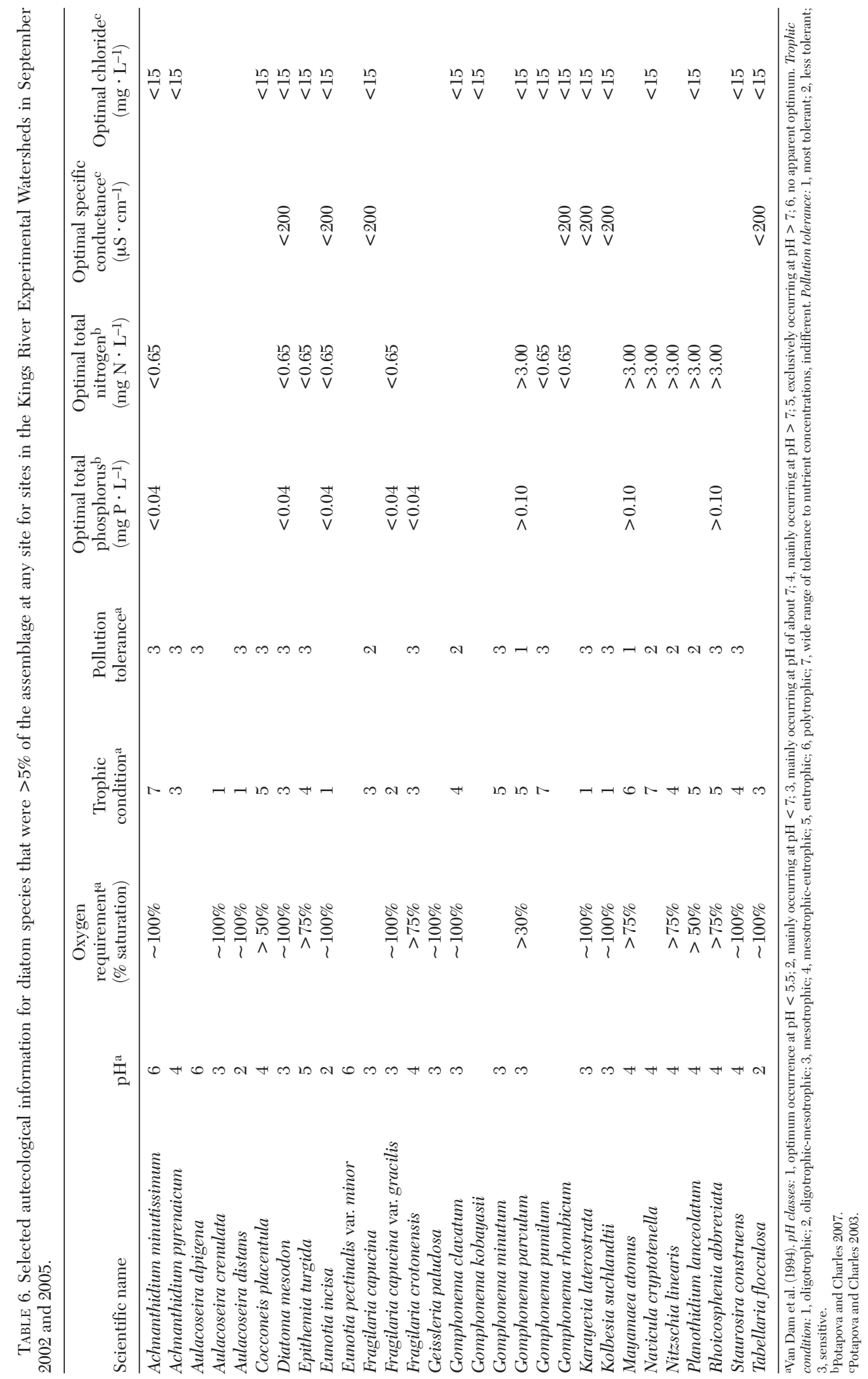


TABLE 7. Discharge $\left(\mathrm{L}^{\cdot} \mathrm{s}^{-1}\right)$ statistics for the 30 days prior to sampling for sites in the Kings River Experimental Watersheds in September 2002 and 2005.

\begin{tabular}{|c|c|c|c|c|c|}
\hline Site & $\begin{array}{c}\text { Mean } \\
\text { discharge }\end{array}$ & $\begin{array}{l}\text { Standard } \\
\text { deviation }\end{array}$ & $\begin{array}{l}\text { Coefficient of } \\
\text { variation }(\%)\end{array}$ & $\begin{array}{l}\text { Minimum } \\
\text { discharge }\end{array}$ & $\begin{array}{l}\text { Maximum } \\
\text { discharge }\end{array}$ \\
\hline \multicolumn{6}{|l|}{2002} \\
\hline D102 & 0.88 & 0.32 & 37 & 0.25 & 1.91 \\
\hline P301 & 0.54 & 0.22 & 41 & 0.22 & 1.20 \\
\hline P303a & 1.22 & 0.15 & 12 & 0.93 & 1.66 \\
\hline P304 & 0.77 & 0.07 & 9 & 0.63 & 1.35 \\
\hline \multicolumn{6}{|l|}{2005} \\
\hline B201 & 2.80 & 0.36 & 13 & 2.00 & 3.80 \\
\hline B203 & 5.04 & 0.80 & 16 & 3.60 & 7.50 \\
\hline B204 & 8.52 & 1.61 & 19 & 5.50 & 13.00 \\
\hline D102 & 3.34 & 0.60 & 18 & 2.40 & 5.00 \\
\hline P301 & 3.12 & 0.52 & 17 & 2.20 & 4.60 \\
\hline P303 & 3.19 & 0.53 & 17 & 2.30 & 4.70 \\
\hline P304 & 5.93 & 0.35 & 6 & 5.20 & 6.90 \\
\hline T003 & 34.41 & 3.50 & 10 & 29.50 & 44.20 \\
\hline
\end{tabular}

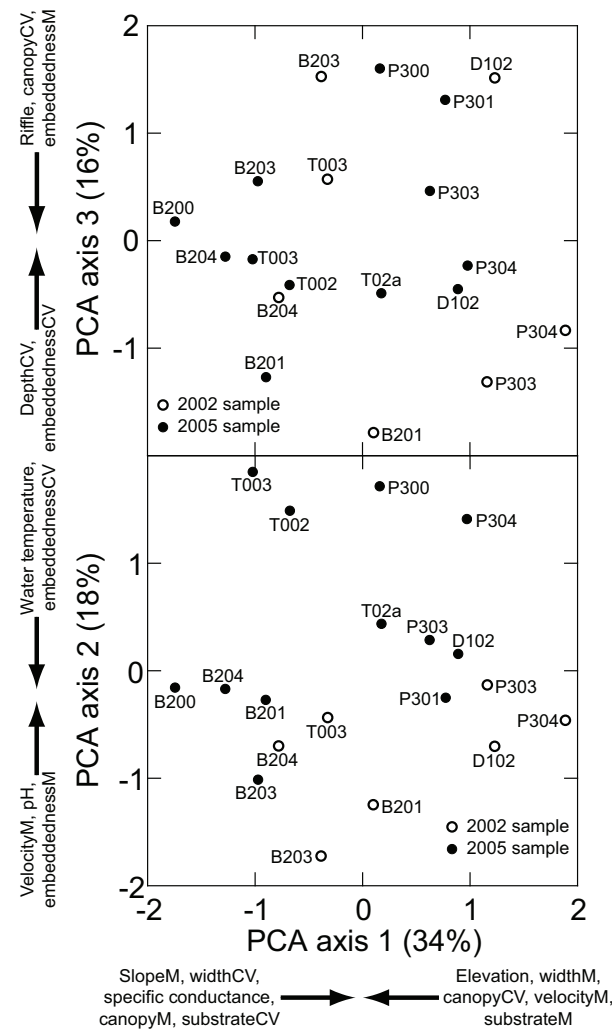

Fig. 3. Plots of site scores for the first 3 axes of a principal components analysis of habitat data collected at sites in the Kings River Experimental Watersheds in September 2002 and 2005. Numbers in parentheses indicate the percentage of total variance explained by each axis. Variables listed next to axes had loadings of greater than 0.5 on that axis. The suffixes $\mathrm{M}$ and $\mathrm{CV}$ denote mean and coefficient of variation for variables with multiple measurements per site. Arrows indicate direction of increasing values. See Figure 1 for site locations.
Creek site T003 was substantially larger than any other gaged site. Variability in discharge was relatively low, and there was no indication of substantial spates that would have scoured algae from the substratum.

The only habitat variable correlated with log-transformed chlorophyll- $a$ data was elevation $(r=-0.53, P<0.02)$. The significant correlation was primarily a result of lower concentrations in all Bull Creek samples compared with Duff Creek samples.

The BV-STEP procedure identified a model including elevation, water temperature, $\mathrm{pH}$, specific conductance, and canopy as the best model explaining diatom assemblage species composition in 81 of 100 tests. A model with mean substratum size rather than specific conductance was selected the other 19 times. The Spearman correlation between habitat and biological data for each model was 0.64 . These variables were associated with the first 2 axes of the principal components analysis (Fig. 3).

\section{Discussion}

Our study is one of only a few addressing the ecology of benthic algae in Sierra Nevada headwater streams. Previous studies in California have focused on larger streams (Brown and Short 1999) or low-elevation streams (Leland et al. 2001, Pan et al. 2006). Leland and Carter (1984, 1985) worked in a small Sierra Nevada stream, but their studies focused on the effects of experimental manipulations of dissolved copper concentrations on benthic algae rather than the ecology of benthic algae 
in different watersheds. Thus, our results provide a valuable baseline for future studies of benthic algae in Sierra Nevada streams, especially for those interested in using algae as a bioindicator.

Biomass of epilithic periphyton can vary widely in rivers, with values ranging in magnitude from 0.01 to $10,000 \mathrm{mg}$ chlorophyll- $a$. $\mathrm{m}^{-2}$ (Morin and Cattaneo 1992). The KREW sites were toward the low end of this range, but not unusual compared with other streams in the Sierra Nevada or headwater streams of similar size in other areas. The KREW measurements were largely within the range of 0.6 to $9.9 \mathrm{mg} \cdot \mathrm{m}^{-2}$ found for sites in the Merced River (Brown and Short 1999), a somewhat larger Sierra Nevada stream. Shortreed and Stockner (1983) documented a similar range of values $\left(0.7-6.7 \mathrm{mg} \cdot \mathrm{m}^{-2}\right)$ from a small stream in British Columbia. High biomass systems are usually dominated by nondiatom algae such as filamentous green algae (e.g., Cladophora sp.) in streams with high nutrient levels (e.g., Stevenson et al. 2006). The relatively low biomass at KREW sites does not seem unusual for small low-nutrient montane streams.

A variety of factors are known to affect biomass of benthic algae in stream systems, including nutrients (Chetelat et al. 1999, Francouer et al. 1999, Biggs 2000, Stelzer and Lamberti 2001, Stevenson et al. 2006), light (Wellnitz et al. 1996, Mosisch et al. 1999, Kiffney and Bull 2000), and hydrology (Biggs and Hickey 1994, Biggs et al. 1999a, 1999b). However, most of these factors do not seem to apply to differences observed in KREW stream biomass between sites and years. Specific conductance and concentrations of dissolved nutrients were generally low at all sites indicating dilute water chemistry. Similarly, nutrients were rarely detected in the Merced River or tributary streams in Yosemite Valley (Brown and Short 1999) at about 1200 m elevation. Canopy (shade) was extensive at all sites (53\%-93\%) and there was not a significant correlation between canopy and chlorophyll- $a$, indicating that light was not a significant factor. There was a significant correlation of chlorophyll- $a$ with elevation. It is unlikely that elevation itself affects benthic algae, but this relationship emphasizes that the Bull Creek sites (the highest sites) were different from the others, with the lowest biomass. The Bull Creek system has a northern exposure and is the most snow-dominated of the watersheds. Snow remains in the Bull Creek watershed from 2 to 4 weeks longer than the other watersheds. Thus, the Bull Creek sites may simply lag behind the other sites in summertime biomass accrual. The PCA analysis also indicated that the Bull Creek sites represent a distinctive combination of habitat conditions compared to the other sites (e.g., higher elevation, wider and more variable canopy, higher velocity, and larger substrata), which might affect biomass accrual. The differences between years are almost certainly due to the much higher flows in 2005 . High water velocities are well known to affect benthic algae biomass (Biggs and Hickey 1994, Biggs et al. 1999a, 1999b, Francouer and Biggs 2006).

Small alpine and montane streams without blooms of filamentous green algae are generally dominated by diatoms and cyanobacteria (Leland and Carter 1984, Brown and Short 1999, Valivova and Lewis 1999). Cyanobacterial cells are generally small compared to diatoms (Lowe and Pan 1996, Academy of Natural Sciences Philadelphia 2006), so high numerical abundance does not necessarily translate to dominance of biomass (Brown and Short 1999, Valivova and Lewis 1999). In general, the KREW streams were dominated by diatoms, by number and probably by biomass.

Diatom species richness in the KREW streams (15-47 species) was generally comparable to other studies of western montane habitats. Brown and Short (1999) found 6-38 diatom species in samples from the Merced River, a Sierra Nevada river. Diatom richness in 2 coastal Oregon watersheds appeared to be slightly lower than in the KREW sites (ranges of 13-30 and 7-23; Naymik and Pan 2005). Similarly, diatom species richness varied from 10 to 26 (median $=15)$ in wadeable Oregon Coast Range streams (Weilhoefer and Pan 2006). Diatom richness in western lowland systems is somewhat higher. Pan et al. (2006) found 7 to 76 species (mean of 41) in lowland California streams. In streams of the Willamette Valley, Oregon, diatom species richness varied from 12 to 50 species (average $=35$; Pan et al. 2004). Sites on the lower-elevation portion of the Truckee River in Nevada, an eastside Sierra Nevada stream, had 16 to 72 species of diatoms (average $=42$; Rushforth and Brock 1991). Overall, the KREW sites do not appear to be particularly depauperate or diverse compared with other western streams. 
Of the species with autecological information available, all were indicative of streams with abundant dissolved oxygen, little organic pollution, and dilute water chemistry (low specific conductance and low concentrations of dissolved chloride). The observation that $\mathrm{pH}$ optima varied widely among the taxa suggests that stream acidification was not a major problem in these drainages because $\mathrm{pH}$-sensitive taxa would disappear and acid-tolerant taxa would dominate the assemblage. This inference is consistent with studies of Sierra Nevada lakes that indicate no change or very slight changes in $\mathrm{pH}$ over the last $60-250$ years (Whiting et al. 1989). The mixture of nutrient optima and species associated with different trophic conditions gives no clear indication regarding nutrient conditions in the KREW streams.

The separation of sites in the NMS ordination was largely associated with the abundances of 5 species. NMS axis 1 represented a gradient of decreasing abundance of Achnanthidium and increasing Cocconeis placentula and Planothidium lanceolatum. Achnanthidium minutissimum and Cocconeis placentula are both considered pioneer species and reach high abundances in recently disturbed streams (Biggs et al. 1998). Weilhoefer and Pan (2006) found, similar to our results, that the abundances of the 2 species were negatively correlated with each other. Natural physical disturbance (e.g., spates) may be a common feature of all of these small headwater streams. One hypothesis is that the relative success of the 2 species is determined by environmental conditions after physical disturbances like spates reduce them to low levels. Since both Cocconeis placentula and Planothidium lanceolatum are considered more eutrophic species (Table 6), NMS axis 1 might represent a subtle response to nutrient conditions. There is no autecological information available to infer the importance of the correlations of the 2 Eunotia species with the 2nd NMS axis.

The BV-STEP procedure showed clear correlations between habitat and species composition of diatom assemblages. As already noted for chlorophyll- $a$, the strong correlation with elevation is more likely related to watershed exposure than to elevation itself. Elevation is also associated with a number of other variables (Fig. 3). The PCA results (Fig. 3) suggest that the Bull Creek watershed represents a distinctive habitat that appears to favor a distinctive assemblage of diatoms.

Our results provide a valuable baseline for future studies of benthic algae in Sierra Nevada headwater streams. The KREW watersheds have been relatively undisturbed by human activities for many years and may serve as useful benchmarks of least-impacted ecological conditions. Our data clearly show that benthic algae are responsive to habitat conditions even within this group of least-impacted sites. Many of the habitat variables we measured can be expected to change in response to human activities, suggesting that benthic algae can serve as a useful bioindicator for monitoring these streams. Thus, depending on the habitat effects of the different forest restoration management strategies being tested in the KREW project, studies of benthic algae will be valuable in determining the effects of management on headwater stream environments.

\section{ACKNOWLEDGMEnTS}

We thank Dave Herbst and numerous USDA Forest Service technicians who aided in the collection of habitat data. Comments from colleagues and journal reviewers significantly improved the quality of the manuscript. This work was supported by interagency agreements with Pacific Southwest Research Station of the USDA Forest Service and the California Bay-Delta Program, Watershed Program Grant 04-186-555-0 to the USDA Forest Service.

\section{Literature Cited}

ACademy of Natural Sciences of Philadelphia. 2006. Autecology of freshwater algae: datafiles and links to internet resources [accessed 1 August 2006]. Philadelphia Academy of Natural Sciences, Phycology Section, Patrick Center for Environmental Research. Available from: http://diatom.acnatsci.org/autecology/.

BigGS, B.J.F. 2000. Eutrophication of streams and rivers: dissolved nutrient-chlorophyll relationships for benthic algae. Journal of the North American Benthological Society 19:17-31.

BiggS, B.J.F., AND C.W. Hickey. 1994. Periphyton responses to a hydraulic gradient in a regulated river in New Zealand. Freshwater Biology 32:49-59.

Biggs, B.J.F., R.A. Smith, AND M.J. DunCan. 1999a. Velocity and sediment disturbance of periphyton in headwater streams: biomass and metabolism. Journal of the North American Benthological Society 18:222-241.

Biggs, B.J.F., J. Stevenson, And R.L. Lowe. 1998. A habitat matrix conceptual model for stream periphyton. Archive für Hydrobiologie 143:21-56. 
Biggs, B.J.F., N.C. Tuchman, R.L. Lowe, and R.J. STEVEnson. 1999b. Resource stress alters hydrological disturbance effects in a stream periphyton community. Oikos 85:95-108.

Bloom, A.M., K.A. Moser, D.F. Porinchu, and G.M. MCDonald. 2003. Diatom-inference models for surface-water temperature and salinity developed from a 57-lake calibration set from the Sierra Nevada, California, USA. Journal of Paleolimnology 29:235255 .

Brown, L.R., ANd T.M. Short. 1999. Biological, habitat, and water quality conditions in the upper Merced River drainage, Yosemite National Park, 1993-1996. U.S. Geological Survey Water-Resources Investigation Report, 99-4088.

Carpenter, K.D., and I.R. Waite. 2000. Relations of habitat-specific algal assemblages to land use and water chemistry in the Willamette Basin, Oregon. Environmental Monitoring and Assessment 64:247-257.

Chetelat, J., F.R. Pick, A. Morin, and P.B. Hamilton. 1999. Periphyton biomass and community composition in rivers of different nutrient status. Canadian Journal of Fisheries and Aquatic Sciences 56:560 569.

Clarke, K.R., AND R.M. WaRWICK. 2001. Change in marine communities: an approach to statistical analysis and interpretation. 2nd edition. Primer-E, Plymouth, U.K.

Francoeur, S.N., AND B.J.F. Biggs. 2006. Short-term effects of elevated velocity and sediment abrasion on benthic algal communities. Hydrobiologia 516:59-69.

Francoeur, S.N., B.J.F. Biggs, R.A. Smith, and R.L. Lowe. 1999. Nutrient limitation of algal biomass accrual in streams: seasonal patterns and a comparison of methods. Journal of the North American Benthological Society 18:242-260.

Hautman, D.P., D. Munch, J.D. Pfaff. 1997. U.S. EPA Method 300.1, determination of inorganic anions in drinking water by ion chromatography. U.S. Environmental Protection Agency, Office of Groundwater and Surface Water, Washington DC.

Hill, B.H., A.T. Herlihy, P.R. Kaufmann, R.J. Stevenson, F.H. MCCormick, AND C.B. Johnson. 2000. Use of periphyton data as an index of biotic integrity. Journal of North American Benthological Society 19:50-67.

Hunsaker, C.T., AND S.M. Eagan. 2003. Small stream ecosystem variability in the Sierra Nevada of California. Pages 716-721 in K.G. Renard, S.A. McElroy, W.J. Gburek, H.E. Canfield, and R.L. Scott, editors, First Interagency Conference on Research in the Watersheds, October 27-30, 2003, U.S. Department of Agriculture, Agricultural Research Service.

Kiffney, P.M., AND J.P. BULl. 2000. Factors controlling periphyton accrual during summer in headwater streams of southwestern British Columbia, Canada. Journal of Freshwater Ecology 15:339-351.

KutKa, FJ., AND C. RICHARDS. 1996. Relating diatom assemblage structure to stream habitat quality. Journal of the North American Benthological Society 15:469480.

LELAND, H.V. 1995. Distribution of phytobenthos in the Yakima River basin, Washington, in relation to geology, land use, and other environmental factors. Canadian Journal of Fisheries and Aquatic Sciences 52:1108-1129.

Leland, H.V., and J.L. Carter. 1984. Effects of copper on species composition of periphyton in a Sierra
Nevada, California, stream. Freshwater Biology 14: 281-296.

1985. Effects of copper on production of periphyton, nitrogen fixation, and processing of leaf litter in a Sierra Nevada, California, stream. Freshwater Biology 15:155-173.

Leland, H.V., and S.D. Porter. 2000. Distribution of benthic algae in the upper Illinois River basin in relation to geology and land use. Freshwater Biology 44:279-301.

Leland, H.V., L.R. Brown, and D.K. Mueller. 2001. Distribution of algae in the San Joaquin River, California, in relation to nutrient supply, salinity, and other environmental factors. Freshwater Biology 46: 1139-1167.

LowE, R.L. 2003. Keeled and canalled raphid diatoms. Page 669-684 in J.D. Wehr and R.G. Sheath, editors, Freshwater algae of North America: ecology and classification. Academic Press, San Diego, CA.

Lowe, R.L., AND Y. Pan. 1996. Benthic algal communities as biological monitors. Pages 705-739 in R.J. Stevenson, M.L. Bothwell, and R.L. Lowe, editors, Algal ecology: freshwater benthic ecosystems. Academic Press, San Diego, CA.

McCormick, P.V., And J. Cairns, JR. 1994. Algae as indicators of environmental change. Journal of Applied Phycology 6:509-526.

Morin, A., and A. Cattaneo. 1992. Factors affecting sampling variability of freshwater periphyton and the power of periphyton studies. Canadian Journal of Fisheries and Aquatic Sciences 49:1198-1205.

Mosisch, T.D., S.E. Bunn, P.M. Davies, and C.J. MarSHALL. 1999. Effects of shade and nutrient manipulation of periphyton growth in a subtropical stream. Aquatic Botany 64:167-177.

Moulton, S.R., II, J.G. Kennen, R.M. Goldstein, And J.A. Намввоок. 2002. Revised protocols for sampling algal, invertebrate, and fish communities as part of National Water-Quality Assessment Program. U.S. Geological Survey, Open File Report 02-150. Reston, VA. 87 pp.

NAYMiK, J., AND Y. PAN. 2005. Diatom assemblages as indicators of timber harvest effects in coastal Oregon streams. Journal of the North American Benthological Society 24:569-584.

O’Dell, J.W., J.D. Pfaff, and W.L. Budde. 1993. Methods for the determination of inorganic substances in environmental samples. U.S. Environmental Protection Agency, Chemistry Research Division, Washington, DC.

Pan, Y., A. Herlihy, P. Kaufmann, J. Wigington, J. van Sickle, AND T. Moser. 2004. Linkages among landuse, water quality, physical habitat conditions and lotic diatom assemblages: a multi-spatial scale assessment. Hydrobiologia 515:59-73.

Pan, Y., B.H. Hill, P. Husby, R.K. Hall, and P.R. KaufMANN. 2006. Relationships between environmental variables and benthic diatom assemblages in California Central Valley streams (USA). Hydrobiologia 516: 119-130.

Pan, Y., R.J. Stevenson, B.H. Hill, A.T. Herlihy, and G.B. Collins. 1996. Using diatoms as indicators of ecological conditions in lotic systems: a regional assessment. Journal of the North American Benthological Society 15:481-495. 
Patrick, R., And C. W. Reimer. 1966. The diatoms of the United States. Monograph 13, Volume 1. Academy of Natural Sciences of Philadelphia. 688 pp.

Potapova, M., AND D.F. Charles. 2003. Distribution of benthic diatoms in U.S. rivers in relation to conductivity and ionic composition. Freshwater Biology 48: 1311-1328.

2007. Diatom metrics for monitoring eutrophication in rivers of the United States. Ecological Indicators $7: 48-70$.

PorTER, S.D. 2008. Algal attributes: an autecological classification of algal taxa collected by the National Water-Quality Assessment Program. U.S. Geological Survey Data Series 329. Available from: http://pubs .usgs.gov/ds/ds329/.

RotT, E., HC. Duthie, And E. Pip. 1998. Monitoring organic pollution and eutrophication in the Grand River, Ontario, by means of diatoms. Canadian Journal of Fisheries and Aquatic Sciences 55:1443-1453.

Rushforth, S.R., AND J.T. Brock. 1991. Attached diatom communities of the lower Truckee River, summer and fall, 1986. Hydrobiologia 224:49-64.

Shortreed, K.S., AND J.G. Stockner. 1983. Periphyton biomass and species composition in a coastal rainforest stream in British Columbia: effects of environmental changes caused by logging. Canadian Journal of Fisheries and Aquatic Sciences 40:1887-1895.

Sonneman, J.A., C.J. Walsh, P.F. Breen, and A.K. Sharpe. 2001. Effects of urbanization on streams of the Melbourne region, Victoria, Australia. II. Benthic diatom communities. Freshwater Biology 46:553-565.

Stelzer, R.S., and G.A. Lamberti. 2001. Effects of N:P ratio and total nutrient concentration on stream peri- phyton community structure, biomass, and elemental composition. Limnology and Oceanography 46:357367.

Stevenson, R.J., S.T. Rier, C.M. Riseng, R.E. Schultz, AND M.J. WiLEy. 2006. Comparing effects of nutrients on algal biomass in streams in two regions with different disturbance regimes and with applications for developing nutrient criteria. Hydrobiologia 561: 149-165.

Van Dam, H., A. Mertens, and J. Sinkeldam. 1994. A coded checklist and ecological indicator values of freshwater diatoms from the Netherlands. Netherlands Journal of Aquatic Ecology 28:117-133.

VAVILOVA, V.V., AND W.M. LEWIS, JR. 1999. Temporal and altitudinal variations in the attached algae of mountain streams in Colorado. Hydrobiologia 390:99-106.

Weilhoefer, C.L., AND Y. PAN. 2006. Diatom assemblages and their associations with environmental variables in Oregon Coast Range streams, USA. Hydrobiologia 561:207-219.

Wellnitz, T.A., R.B. Rader, And J.V. WARD. 1996. Importance of light and nutrients in structuring an algal community in a rocky mountain stream. Journal of Freshwater Ecology 11:399-413.

Wetzel, R.G., And G.E. Likens. 1978. Limnological analyses. W.B. Saunders Company, Philadelphia, PA. Whiting, M.C., D.R. Whitehead, R.W. Holmes, and S.A. Norton. 1989. Paleolimnological reconstruction of recent acidity changes in four Sierra Nevada lakes. Journal of Paleolimnology 2:285-304.

Received 18 April 2007 Accepted 29 October 2007 